\title{
The Hepatoprotective Effect of Bupleurum kaoi, an Endemic Plant to Taiwan, against Dimethylnitrosamine-Induced Hepatic Fibrosis in Rats
}

\author{
Ming-Hong Yen, ${ }^{a}$ Ting-Chun Weng, ${ }^{a}$ Sin-Yie Liu, ${ }^{b}$ Chee-Yin ChaI,${ }^{c}$ and Chun-Ching Lin ${ }^{*}, a$ \\ ${ }^{a}$ Graduate Institute of Natural Products, College of Pharmacy, Kaohsiung Medical University; Kaohsiung, Taiwan, \\ R.O.C.: ${ }^{b}$ Senior Agronomist, Agronomy Division, Agricultural Research Institute, Council of Agriculture; Executive Yuan, \\ Taiwan, R.O.C.: and ${ }^{c}$ Department of Pathology, College of Medicine, Kaohsiung Medical University; Kaohsiung, Taiwan, \\ R.O.C. Received April 21, 2004; accepted November 24, 2004
}

In the present study, three materials extracted or isolated from the roots of $\mathrm{B}$. $\mathrm{kaoi}$, an endemic plant to Taiwan, were used to be examined the hepatoprotective effect against dimethylnitrosamine (DMN)-induced hepatic fibrosis in rats, they were water extract (BKW), polysaccharide-enriched fractions (BKP) and saponin-enriched fractions (BKS). After treated with DMN for 4 weeks, the levels of aminotrasferases (GOT, GPT) were significantly elevated in serum, and the levels of total protein (TP) and albumin were significantly decreased in serum and liver homogenates. Furthermore, the collagen contents were significantly elevated in liver homogenates and corresponded to the hepatofibrotic pathological examination. As the results showed, treated with groups of BKW, BKP, BKS markedly reduced GOT, GPT levels in rats serum. In addition, treated with groups of BKW, BKP, BKS markedly raised TP levels in rats serum and liver homogenates. Furthermore, treated with groups of BKW, BKP markedly raised albumin levels in rats serum and liver homogenates. Treated with groups of BKW, BKP, BKS markedly raised interferon- $\gamma($ IFN- $\gamma$ ) levels in rats serum, where only BKS and silymarin markedly raised interkeukin-10 (IL-10) levels in rats serum compared to that of DMN treated rats. None of test materials of $B$. kaoi except silymarin reduced the malondialdehyde (MDA) levels, but BKW, BKP markedly raised hepatic glutathione (GSH) levels to reveal the activity of anti-lipid peroxidation. Otherwise, treated with groups of BKW, BKP, BKS significantly reduced collagen contents in rats liver homogenates. In conclusion, B. kaoi demonstrated the anti-inflammatory and anti-fibrotic activities followed by anti-oxidant activity of enhanced GSH production, enhanced the liver cell regeneration and concerned with regulations of INF- $\gamma$ and IL-10. The ability of hepatoprotective and anti-fibrotic activities of $B$. kaoi are higher than B. chinense, a Bupleuri Radix imported from China to Taiwan.

Key words Bupleurum kaoi; Bupleurum chinense; polysaccharide-enriched fraction; saponin-enriched fraction; dimethylnitrosamine; hepatic fibrosis

Bupleuri Radix (Chai-hu in Chinese and Saiko in Japanese) is one of the most important traditional Chinese medicines for treating hepatitis, jaundice, bitter taste in the mouth, dizziness, lung disease and delayed menstruation. According to herbological study, the sources of Bupleuri Radix were originally derived from the roots of Bupleurum spp. (Family: Umbelliferae), especially $B$. chinense from mainland China and $B$. falcatum from Japan. Many investigations indicated that $B$. chinense as well as $B$. falcatum exhibited a potent hepatoprotective effects against chemicals induced hepatotoxicity such as carbon tetrachloride $\left(\mathrm{CCl}_{4}\right)$, acetaminophen, $\beta$-Dgalactosamine (D-GalN), respectively. ${ }^{1-4)}$ Furthermore, Shosaiko-to was well known as a valuable herb prescriptions of Chinese medicines widely used in the treatment of chronic liver diseases, including chronic hepatic inflammation, fibrosis, and viral hepatitis. Recently, many investigations have been previously reported that Sho-saiko-to exhibited the liver fibrosis improving effect and due to the ability of the inhibition of activated myofibroblasts activation and enhancing liver cell regeneration. ${ }^{5-7)}$ In addition, Sho-saiko-to was prepared from seven herbs and the major herb was Bupleuri Radix. Therefore, it was very interested for us to developing the new resources of Bupleuri Radix in Taiwan.

Recently, B. kaio Liu, Chau et Chuang, an endemic plant to Taiwan, has been locally used as a hepatoprotective herb for few years in Taiwan. We have previously investigated that B. kaoi exhibited a hepatoprotective effect against $\mathrm{CCl}_{4}$ and D-GalN induced acute hepatic injury in rats. The results showed that $B$. kaio enhanced liver cell regeneration and re- duced inflammatory infiltration in rat liver to improve the hepatic injury induced by $\mathrm{CCl}_{4}$ or D-GalN intoxication. ${ }^{8,9)} \mathrm{We}$ were further determined the quantities of saikosaponins-a, c, $\mathrm{d}$ in different Bupleuri Radix which saikosaponins were the major active components of Bupleuri Radix. The results showed B. kaio has greater quantities of saikosaponins-a, c, d than B. falcatum and B. chinense. ${ }^{10)}$ In the present study, we used dimethylnitrosamine (DMN) to induce hepatic fibrosis in rats and examined the hepatoprotective effects of $B$. kaoi as well as $B$. chinense. DMN was a famous hepatotoxicant frequently used to experimentally induce liver fibrosis due to its convenience. ${ }^{11)}$

Subsequently, three materials extracted or isolated from the roots of $B$. kaoi and B. chinense were used to be examined in the present study, they were water extract (BKW for $B$. kaoi, $\mathrm{BCW}$ for $B$. chinense), polysaccharide-enriched fractions (BKP for $B$. kaoi, BCP for B. chinense) and saponin-enriched fractions (BKS for $B$. kaoi, BCS for B. chinense). The liver inflammation and hepatofibrosis-improving effects were evaluated according to GOT, GPT (serum biochemical indicators for liver inflammation), albumin, total protein (liver cell regeneration indicators), IFN- $\gamma$ and IL-10 (immune response of anti-inflammation), GSH and MDA (anti-oxidant indicators), collagen contents (fibrotic indicators) as well as histopathological examination of rats liver.

\section{MATERIALS AND METHODS}

Animals Male Sprague-Dawley rats (aged 4-6 weeks) 
weighing $130-150 \mathrm{~g}$ were purchased from Animal Center of NSC, Taipei, Taiwan, R.O.C. Animals were housed in airconditioned room at $22 \pm 3{ }^{\circ} \mathrm{C}, 55-60 \%$ relative humidity and a $12 \mathrm{~h}$ light/dark cycle and fed with a standard laboratory diet and tap water throughout the experiment. Animals were acclimatized for 1 week and 8 animals assigned to each group.

Chemicals 2,4-Dinitrophenylhydrazine (DNPH), butylated hydroxytoluene (BHT), 1,1,3,3,-tetra-ethoxypropane (TEP), silymarin and DMN were purchased from Sigma Chemical Co., U.S.A. Sirius red F3B (NO.34149) was purchased from Gurr BDH Chemicals Ltd., Poole, England. All of other chemicals were of reagent grade and used as received.

Plant Materials The roots of $B$. kaoi was purchased from Green Health Biotechnology Co., Ltd. (Yuanlin, Taiwan) and $B$. chinense was purchased from Koda Pharmaceutical Co., Ltd. (Chunle, Taiwan). The two plant materials were authenticated by Professor Lin C. C., Kaohsiung Medical University, Kaohsiung, Taiwan, R.O.C., with anatomical examination.

Water Extracts Preparation Plant materials were dried, chopped, and decocted with 10-fold volume of boiled $d-\mathrm{H}_{2} \mathrm{O}$ for three times. Each duration of decoction time was $1 \mathrm{~h}$. The filtrations were combined and centrifuged (1700 g, $10 \mathrm{~min})$. The supernatant was concentrated by rotary evaporator under vacuum, and then lyophilized to obtain dried power. Yield BKW was $15.4 \%$ and BCW was $17.2 \%$, respectively.

Polysaccharide-Enriched Fractions Preperation One hundred grams water extracts of each of $B$. kaoi and B. chinense was refluxed 5 times with $31 \mathrm{MeOH}$ for $1 \mathrm{~h}$ and centrifuged $(1700 \mathrm{~g}, 10 \mathrm{~min})$. After discard supernatant, the residues was dissolved in $\mathrm{H}_{2} \mathrm{O}$ and then 5-folds volume of EtOH was added. The resulting precipitants was redissolved in $\mathrm{H}_{2} \mathrm{O}$ and dialyzed against running $\mathrm{H}_{2} \mathrm{O}$ for $3 \mathrm{~d}$. The nondialysable portion was centrifuged to remove $\mathrm{H}_{2} \mathrm{O}$-insoluble material and the supernatant was lyophilized. The yield of BKP is $7.14 \%$ and BCP is $7.51 \%$, respectively.

Saponin-Enriched Fractions Preparation Plant materials were dried, chopped, and extracted with 10-fold volume of methanol for three times under reflux. Each duration of extraction time was $1 \mathrm{~h}$. The methanol filtrations were evaporated to dry. The residue was suspended in $d-\mathrm{H}_{2} \mathrm{O}$ and subsequently partitioned with $n-\mathrm{BuOH}$. The organic layer was concentrated in vacuo, then the residue was dissolved in methanol and precipitated in ethyl ether to obtained the saponin-enriched fractions. The yield of BKP is $3.76 \%$ and BCP is $2.21 \%$, respectively.

Experimental Design Hepatic fibrosis model in rats was produced by admimistration of DMN. After acclimatization of 1 week, DMN $(10 \mathrm{mg} / \mathrm{kg}$ per day for 3 consecutive days of each week for 4 weeks) was injected i.p. to induce hepatic fibrosis in rats according to the Weng H. L. et al., described. ${ }^{12)}$ After treated with DMN 2 weeks, animals were administed orally with $25 \mathrm{mg} / \mathrm{kg}$ silymarin, $100 \mathrm{mg} / \mathrm{kg} \mathrm{BKW}$ and BCW, $50 \mathrm{mg} / \mathrm{kg} \mathrm{BKP}$ and $\mathrm{BCP}, 50 \mathrm{mg} / \mathrm{kg} \mathrm{BKS}$ and $\mathrm{BCS}$, respectively, twice a day for the last two weeks. At the 29th day after experimental periods, all animals were sacrificed and blood was collected from the carotid artery. Blood samples were allowed to coagulate at $4{ }^{\circ} \mathrm{C}$ for $30 \mathrm{~min}$. Serum was then separated by centrifugation at $4{ }^{\circ} \mathrm{C}, 3000 \mathrm{rpm}$ for $10 \mathrm{~min}$.
Each of rats liver was removed immediately after blood was collected. Each of middle lobe livers was homogenized in 9fold volume of ice-cold $1.15 \% \mathrm{KCl}$ to obatain the liver homogenates. Each of other lobes livers was fixed in 10\% neutral formalin and processed for paraffin embedding. Liver tissues were further examined with histopathological observation.

Assessment of Liver Functions The levels of GOT, GPT, total protein (TP), albumin, triglyceride (TG) in serum and the levels of TP, albumin in liver homogenates were measured using a Ciba-Corning 550E analyzer (Global Medical Instrumentation Inc., U.S.A.).

Assessment of IL-10 and IFN- $\boldsymbol{\gamma}$ The concentrations of IL-10 and INF- $\gamma$ in serum were depended using commercial ELISA kits (P\&D Systems Inc., U.S.A.) and followed the manufacturer's recommendations.

Assessment of Malondialdehyde Levels The malondialdehyde (MDA) levels in liver homogenates were determined following the method of Kawai et al., described. ${ }^{13)}$ In this method, HPLC system was used and TEP as a standard finally.

Assessment of Glutathione Levels The total hepatic reduced glutathione (GSH) concentration was determined by the method of Tietze. ${ }^{14)}$ Each of liver tissues was homogenized with phosphate buffer $(\mathrm{pH} 7.5)$ containing $10 \mathrm{~mm}$ EDTA and centrifuged at $4{ }^{\circ} \mathrm{C}, 10000 \mathrm{~g}$ for $10 \mathrm{~min}$. The supernatant was used for the total glutathione concentration assay.

Assessment of Collagen Levels The method was performed according to the method of Jimenez W. et al. ${ }^{15}$ ) Briefly, a $15 \mu \mathrm{m}$-thick liver sections were placed on slides. After de-paraffinized, the liver sections were firstly stained with a saturated picric acid in distilled water contain $0.01 \%$ of Fast green FCF followed by stained with picric acid containing $0.04 \%$ of Fast green and $0.1 \%$ of Sinus red F3B. After incubated in the dark at room temperature for $30 \mathrm{~min}$, the samples were transferred to a test tube containing $1 \mathrm{ml}$ of $0.1 \% \mathrm{NaOH}$ in absolute methanol $(1: 1, \mathrm{v} / \mathrm{v})$. The tubes were gently mixed until the color was eluted completely. Absorbance of the eluted color was read in a U-2001 spectrophotometer (Hitachi Co., Japan). Fast green has its maximal absorbance at $630 \mathrm{~nm}$ and Sinus red at $540 \mathrm{~nm}$. The collagen contents were calculated as followed:

$$
\begin{aligned}
& \text { non-collagenous protein }(\mathrm{mg})=\frac{\text { absorbance } 605 \mathrm{~nm}}{2.08} \\
& \text { collagen }(\mu \mathrm{g})=\frac{\text { absorbance } 540 \mathrm{~nm}-0.26 \text { absorbance } 605 \mathrm{~nm}}{38.4} \\
& \text { collagen content }(\mu \mathrm{g} / \mathrm{mg} \text { total protein }) \\
& =\frac{\mu \mathrm{g} \text { collagen }}{\mu \mathrm{g} \text { collagen }+\mathrm{mg} \text { non-collagenous protein }}
\end{aligned}
$$

Histopathological Examination The paraffin embedded liver tissues were sliced into $2-3 \mu \mathrm{m}$ pieces and stained with Hematoxylin-Eosin (H-E) and Masson-Trichrome (M-T), respectively, for photomicroscopic assessment.

Statistical Analysis All values were expressed as the mean \pm S.D. $(n=6)$. Significant differences between the groups were statistically analyzed using an one-way analysis of variance (ANOVA), followed by a two pairs Student's $t$ test. $p<0.05$ or less was considered statistically significant. 


\section{RESULTS}

Effect of Body Weight in Rats As Table 1 shows, after treated with DMN in period of $28 \mathrm{~d}$, rats body weight of DMN treated group was markedly decreased $(p<0.01 \mathrm{com}-$ pared to that of normal control group). Furthermore, the rats body weight were significantly elevated when treated with all test materials and silymarin, except BCS of $B$. chinense, compared to that of DMN treated group.

Effect of Liver Function Examination As Table 2 shows, the GOT (147.5 $\pm 6.47 \mathrm{IU} / \mathrm{l})$, GPT $(109.2 \pm 14.41 \mathrm{IU} / \mathrm{l})$ and triglyceride (TG; $105.0 \pm 8.6 \mathrm{mg} / \mathrm{dl}$ ) levels were significantly elevated after DMN treatment compared to normal control group (GOT: 101.0 \pm 6.68 , GPT: $39.3 \pm 4.27$, TG: $66.0 \pm 20.9$, respectively). In contrast, treated with all plant materials and silymarin, except BCS of $B$. chinense, markedly reduced enzyme activities compared to that of DMN treated group.

Furthermore, the levels of TP, albumin in serum and liver homogenates, respectively, were significantly decreased in DMN treated group as Table 3 showed $(p<0.001$ in serum and $p<0.01$ in liver homogenates, respectively, compared to that of normal control group). In contrast, treated with BKW, BKP, BKS of B. kaoi markedly raised the levels of TP in serum and liver homogenates. In $B$. chinense groups, only BCP markedly raised the level of TP in liver homogenates. On the other hand, treated with BKW, BKP of B. kaoi and $\mathrm{BCW}$ of $B$. chinense markedly raised the level of albumin in serum, and $\mathrm{BKW}, \mathrm{BKP}$ of $B . k a o i$ and $\mathrm{BCP}$ of $B$. chinense markedly raised the level of albumin in liver homogenates. According to these results, B. kaoi showed more potent hepatoprotective effect than $B$. chinense (Newman-Keuls test).

Effects of Cytokines Production As Table 4 shows, the levels of IL-10 was significantly decreased in DMN treated group ( $p<0.001$ compared to that of normal control group). Only BKS of $B$. kaoi and silymarin significantly elevated the level of IL-10. Furthermore, the level of IFN- $\gamma$ was significantly increased in DMN treated group $(p<0.05$ compared to that of normal control group). As except, BKW, BKP, BKS of $B . k a o i$ and $\mathrm{BCW}$ of $B$. chinense significantly enhanced the IFN- $\gamma$ production compared to that of DMN treated group.

Effect of Lipid Peroxidation As Table 5 shows, the level of MDA, a lipid peroxidation indicator, was signifi-

Table 1. Effect of Administrations of B. kaoi and B. chinense Extracts on the Change of Body Weight (g) in Dimethylnitrosamine (DMN)-Induced Liver Injury in Rats

\begin{tabular}{|c|c|c|c|c|}
\hline \multirow{2}{*}{ Groups } & \multirow{2}{*}{$\begin{array}{c}\text { Dose } \\
(\mathrm{mg} / \mathrm{kg})\end{array}$} & \multicolumn{3}{|c|}{ Days after DMN treatment } \\
\hline & & 0 & 14 & 28 \\
\hline Normal control & - & $150.0 \pm 12.2$ & $228.0 \pm 13.3$ & $340.0 \pm 33.9$ \\
\hline DMN & 10 & $143.7 \pm 23.3$ & $225.0 \pm 19.3$ & $265.0 \pm 10.5^{\# \#}$ \\
\hline Silymarin+DMN & 25 & $145.0 \pm 20.0$ & $226.2 \pm 16.9$ & $290.0 \pm 22.0 * *$ \\
\hline \multicolumn{5}{|l|}{ B. chinense roots } \\
\hline $\mathrm{BCW}+\mathrm{DMN}$ & 100 & $145.0 \pm 20.0$ & $226.2 \pm 16.9$ & $288.0 \pm 16.4^{*}$ \\
\hline $\mathrm{BCP}+\mathrm{DMN}$ & 50 & $145.0 \pm 16.9$ & $225.0 \pm 16.0$ & $281.6 \pm 9.8 * *$ \\
\hline $\mathrm{BCS}+\mathrm{DMN}$ & 50 & $145.0 \pm 16.9$ & $225.0 \pm 16.0$ & $277.5 \pm 15.0$ \\
\hline \multicolumn{5}{|l|}{ B. kaoi roots } \\
\hline $\mathrm{BKW}+\mathrm{DMN}$ & 100 & $145.0 \pm 16.0$ & $226.2 \pm 16.9$ & $288.3 \pm 13.3 * *$ \\
\hline $\mathrm{BKP}+\mathrm{DMN}$ & 50 & $143.7 \pm 16.0$ & $227.5 \pm 14.9$ & $285.0 \pm 15.2 *$ \\
\hline $\mathrm{BKS}+\mathrm{DMN}$ & 50 & $138.7 \pm 17.3$ & $226.2 \pm 17.7$ & $297.5 \pm 15.2 * *$ \\
\hline
\end{tabular}

Each value presents the mean \pm S.D. $(n=6)$. DMN $(10 \mathrm{mg} / \mathrm{kg}$ per day for 3 consecutive days of each week for 4 weeks $)$ was injected i.p. to induce hepatic fibrosis in rats. Test drugs were administered orally twice a day for the last 2 weeks. BCW: water extracts; BCP: polysaccharide-enriched fractions; BCS : saponin-enriched fractions which were isolated from the roots of $B$. chinense. BKW: water extracts; BKP: polysaccharide-enriched fractions; BKS: saponin-enriched fractions which were isolated from the roots of $B$. kaoi. \#\# $p<0.01$ significantly different from normal control group; $* p<0.05, * * p<0.01$ significantly different from DMN treated group.

Table 2. Effect of Administrations of B. kaoi and B. chinense Extracts on GOT, GPT, Triglyceride (TG) in Dimethylnitrosamine (DMN)-Induced Liver Injury in Rats

\begin{tabular}{lcccc}
\hline \hline Groups & $\begin{array}{c}\text { Dose } \\
(\mathrm{mg} / \mathrm{kg})\end{array}$ & $\begin{array}{c}\text { GOT } \\
(\mathrm{IU} / \mathrm{l})\end{array}$ & $\begin{array}{c}\text { GPT } \\
(\mathrm{IU} / \mathrm{l})\end{array}$ & $\begin{array}{c}\text { TG } \\
(\mathrm{mg} / \mathrm{dl})\end{array}$ \\
\hline Normal control & - & $101.0 \pm 6.68$ & $39.3 \pm 4.27$ & $66.0 \pm 20.9$ \\
DMN & 10 & $147.5 \pm 6.47^{\# \#}$ & $109.2 \pm 14.41^{* \# \#}$ & $105.0 \pm 8.6^{\# \#}$ \\
Silymarin + DMN & 25 & $158.5 \pm 20.57$ & $79.7 \pm 17.44^{*}$ & $69.0 \pm 19.6^{* *}$ \\
B. chinense roots & & & & \\
BCW+DMN & 100 & $117.7 \pm 8.48^{* * *}$ & $82.0 \pm 11.52^{*}$ & $120.3 \pm 22.5$ \\
BCP+DMN & 50 & $120.2 \pm 14.47^{* *}$ & $79.8 \pm 18.01^{*}$ & $90.5 \pm 15.8^{*}$ \\
BCS+DMN & 50 & $163.8 \pm 23.16$ & $104.8 \pm 24.39$ & $67.5 \pm 12.6^{* *}$ \\
B. $k$ aoi roots & & & & \\
BKW+DMN & 100 & $127.3 \pm 11.69^{* *}$ & $58.8 \pm 9.09^{* * *}$ & $81.7 \pm 4.6^{* *}$ \\
BKP+DMN & 50 & $114.7 \pm 12.44^{* *}$ & $60.8 \pm 9.50^{* * *}$ & $73.7 \pm 13.4^{* *}$ \\
BKS+DMN & 50 & $58.3 \pm 16.85^{* *}$ & $67.7 \pm 15.2^{* *}$
\end{tabular}

Each data was determined in rats serum and each value presents the mean \pm S.D. $(n=6)$. DMN ( $10 \mathrm{mg} / \mathrm{kg}$ per day for 3 consecutive days of each week for 4 weeks) was injected i.p. to induce hepatic fibrosis in rats. Test drugs were administered orally twice a day for the last 2 weeks. BCW: water extracts; BCP: polysaccharide-enriched fractions; BCS: saponin-enriched fractions which were isolated from the roots of $B$. chinense. BKW: water extracts; BKP: polysaccharide-enriched fractions; BKS: saponin-enriched fractions which were isolated from the roots of $B$. kaoi. \# $p<0.01$, \# $p<0.001$ significantly different from normal control group; $* p<0.05$, ** $p<0.01, * * * p<0.001$ significantly different from DMN treated group. 
Table 3. Effect of Administrations of B. kaoi and B. chinense Extracts on Total Protein (TP) and Albumin in Dimethylnitrosamine (DMN)-Induced Liver Injury in Rats

\begin{tabular}{|c|c|c|c|c|c|}
\hline \multirow[b]{2}{*}{ Groups } & \multirow[b]{2}{*}{$\begin{array}{c}\text { Dose } \\
(\mathrm{mg} / \mathrm{kg})\end{array}$} & \multicolumn{2}{|c|}{ Serum } & \multicolumn{2}{|c|}{ Liver homogenates } \\
\hline & & $\begin{array}{c}\mathrm{TP} \\
(\mathrm{g} / \mathrm{dl})\end{array}$ & $\begin{array}{l}\text { Albumin } \\
(\mathrm{g} / \mathrm{dl})\end{array}$ & $\begin{array}{c}\mathrm{TP} \\
(\mathrm{g} / \mathrm{dl})\end{array}$ & $\begin{array}{l}\text { Albumin } \\
(\mathrm{g} / \mathrm{dl})\end{array}$ \\
\hline Normal control & - & $6.83 \pm 0.21$ & $4.24 \pm 0.02$ & $1.160 \pm 0.182$ & $0.845 \pm 0.139$ \\
\hline DMN & 10 & $5.77 \pm 0.19^{\# \# \#}$ & 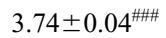 & $0.767 \pm 0.052^{\# \#}$ & $0.502 \pm 0.057^{\# \#}$ \\
\hline Silymarin+DMN & 25 & $6.22 \pm 0.26^{*}$ & $3.77 \pm 0.14$ & $0.800 \pm 0.089$ & $0.593 \pm 0.064 *$ \\
\hline \multicolumn{6}{|l|}{ B. chinense roots } \\
\hline $\mathrm{BCW}+\mathrm{DMN}$ & 100 & $5.93 \pm 0.15$ & $3.87 \pm 0.08 *$ & $0.867 \pm 0.103$ & $0.587 \pm 0.081$ \\
\hline $\mathrm{BCP}+\mathrm{DMN}$ & 50 & $5.83 \pm 0.20$ & $3.82 \pm 0.17$ & $0.900 \pm 0.063 * *$ & $0.630 \pm 0.069 *$ \\
\hline $\mathrm{BCS}+\mathrm{DMN}$ & 50 & $5.80 \pm 0.26$ & $3.70 \pm 0.11$ & $0.875 \pm 0.171$ & $0.605 \pm 0.149$ \\
\hline \multicolumn{6}{|l|}{ B. kaoi roots } \\
\hline $\mathrm{BKW}+\mathrm{DMN}$ & 100 & $6.02 \pm 0.12 *$ & $3.95 \pm 0.08 * *$ & $1.050 \pm 0.105 * *$ & $0.695 \pm 0.097 * *$ \\
\hline $\mathrm{BKP}+\mathrm{DMN}$ & 50 & $6.00 \pm 0.14^{*}$ & $3.90 \pm 0.15^{*}$ & $1.001 \pm 0.167 *$ & $0.677 \pm 0.113 *$ \\
\hline $\mathrm{BKS}+\mathrm{DMN}$ & 50 & $6.23 \pm 0.33^{*}$ & $3.76 \pm 0.16$ & $0.883 \pm 0.098 *$ & $0.572 \pm 0.072$ \\
\hline
\end{tabular}

Each value presents the mean \pm S.D. $(n=6)$. DMN $(10 \mathrm{mg} / \mathrm{kg}$ per day for 3 consecutive days of each week for 4 weeks $)$ was injected i.p. to induce hepatic fibrosis in rats. Test drugs were administered orally twice a day for the last 2 weeks. BCW: water extracts; BCP: polysaccharide-enriched fractions; BCS: saponin-enriched fractions which were isolated from the roots of $B$. chinense. BKW: water extracts; BKP: polysaccharide-enriched fractions; BKS: saponin-enriched fractions which were isolated from the roots of $B$. kaoi. \# $p<0.01$, \# $p<0.001$ significantly different from normal control group; $* p<0.05$, $* * p<0.01$ significantly different from DMN treated group.

Table 4. Effect of Administrations of B. kaoi and B. chinense Extracts on Interferon- $\gamma$ (IFN- $\gamma$ ) and Interleukin-10 (IL-10) in Dimethylnitrosamine (DMN)Induced Liver Injury in Rats

\begin{tabular}{lccc}
\hline \hline Groups & Dose $(\mathrm{mg} / \mathrm{kg})$ & IL-10 $(\mathrm{pg} / \mathrm{ml})$ & IFN- $\gamma(\mathrm{pg} / \mathrm{ml})$ \\
\hline Normal control & - & $128.8 \pm 21.05$ & $81.3 \pm 21.34$ \\
DMN & 10 & $61.2 \pm 25.38^{\# \#}$ & $113.4 \pm 21.52^{\# \#}$ \\
Silymarin+DMN & 25 & $91.7 \pm 20.61^{*}$ & $123.8 \pm 34.04$ \\
B. chinense roots & & & $143.3 \pm 14.41^{*}$ \\
BCW+DMN & 100 & $60.8 \pm 15.39$ & $97.2 \pm 25.29$ \\
BCP+DMN & 50 & $78.5 \pm 26.58$ & $118.5 \pm 34.92$ \\
BCS+DMN & 50 & $61.2 \pm 13.98$ & $181.6 \pm 34.22^{* *}$ \\
B. kaoi roots & 100 & $66.7 \pm 16.86$ & $204.1 \pm 55.60^{* *}$ \\
BKW+DMN & 50 & $57.1 \pm 22.15$ & $174.8 \pm 15.02^{* * *}$ \\
BKP+DMN & 50 & $125.2 \pm 45.91^{* *}$ & \\
BKS+DMN & &
\end{tabular}

Each data was determined in rats serum and each value presents the mean \pm S.D. $(n=6)$. DMN $(10 \mathrm{mg} / \mathrm{kg}$ per day for 3 consecutive days of each week for 4 weeks $)$ was injected i.p. to induce hepatic fibrosis in rats. Test drugs were administered orally twice a day for the last 2 weeks. BCW: water extracts; BCP: polysaccharide-enriched fractions; BCS: saponin-enriched fractions which were isolated from the roots of $B$. chinense. BKW: water extracts; BKP: polysaccharide-enriched fractions; BKS: saponin-enriched fractions which were isolated from the roots of B. kaoi. \# $p<0.001$ significantly different from normal control group; $* p<0.05, * * p<0.01, * * * p<0.001$ significantly different from DMN treated group.

cantly elevated in DMN treated group $(p<0.01$ compared to that of normal control group). Only the reference drug silymarin significantly reduced the levels of MDA in all examined test materials $(p<0.05$ compared to that of DMN treated group).

Effect of Hepatic GSH Levels As Table 5 shows, the GSH $(0.502 \pm 0.057 \mu \mathrm{g} / \mathrm{mg}$ protein) levels were significantly decreased after DMN treatment compared to normal control group $(0.806 \pm 0.149 \mu \mathrm{g} / \mathrm{mg}$ protein $)$. In contrast, treated with BKW, BKP of B. kaoi, BCW, BCP of B. chinense and silymarin, respectively, markedly elevated hepatic GSH levels compared to that of DMN treated group.

Effect of Collagen Contents As Table 5 shows, the collagen content in liver homogenates of DMN treated group $(96.42 \pm 3.16 \mu \mathrm{g} / \mathrm{mg}$ protein) was significantly elevated than normal control group $(83.89 \pm 2.58 \mu \mathrm{g} / \mathrm{mg}$ protein, $p<0.05)$. All test materials and silymarin, except BCS of $B$. chinense, significantly reduced the level of collagen content compared to that of DMN treated group.

Histopathological Examination The histopathological change of hepatic inflammation was examined with $\mathrm{H}-\mathrm{E}$ stain in liver tissues. As Fig. 1 shows, treatment with DMN for 4 weeks showed remarkably extensive necrosis and disruption of tissue architecture around central vein (Zone 3 area) (Fig. 1B). These alterations were slightly or modulatory reduced with silymarin (Fig. 1C) and the two plant materials treatment in rats (Figs. 1D-I).

In addition, the hepatic fibrosis degree was stained with M-S for collagen. As Fig. 2 shows, collagen fibers were slightly existed only in the periportal area in normal control group (Fig. 2A). In contrast, the liver section of the DMN treated rats exhibited an increase of collagen content, and displayed bundles of collagen fibrous surrounding the lobules, forming markedly large fibrous septa (Fig. 2B). The formation and accumulation of collagen fibers were reduced with silymarin (Fig. 2C) and the two plant materials treatment in rats (Figs. 2D-I).

\section{DISCUSSION}

In many chronic liver diseases including viral hepatitis, chronic alcoholic hepatitis, chemical toxicants induced hepatitis, autoimmune disorders can be found hepatic inflammative infiltration, and progressively destroying the liver 
Table 5. Effect of Administrations of B. kaoi and B. chinense Extracts on Glutathione (GSH), Malondialdehyde (MDA), Collagen Contents in Dimethylnitrosamine (DMN)-Induced Liver Injury in Rats

\begin{tabular}{|c|c|c|c|c|}
\hline Groups & $\begin{array}{c}\text { Dose } \\
(\mathrm{mg} / \mathrm{kg})\end{array}$ & $\begin{array}{c}\text { GSH } \\
(\mu \mathrm{g} / \mathrm{mg} \text { protein })\end{array}$ & $\begin{array}{c}\text { MDA } \\
(\mu \mathrm{g} / \mathrm{mg} \text { protein })\end{array}$ & $\begin{array}{l}\text { Collagen contents } \\
(\mu \mathrm{g} / \mathrm{mg} \text { protein })\end{array}$ \\
\hline Normal control & - & $0.806 \pm 0.149$ & $0.039 \pm 0.028$ & $83.89 \pm 2.58$ \\
\hline DMN & 10 & $0.502 \pm 0.057^{\#}$ & $0.330 \pm 0.217^{\# \#}$ & $96.42 \pm 3.16^{\#}$ \\
\hline Silymarin+DMN & 25 & $0.630 \pm 0.069 *$ & $0.203 \pm 0.146^{*}$ & $89.10 \pm 2.55 * *$ \\
\hline \multicolumn{5}{|l|}{ B. chinense roots } \\
\hline $\mathrm{BCW}+\mathrm{DMN}$ & 100 & $0.660 \pm 0.141 *$ & $0.175 \pm 0.087$ & $90.56 \pm 3.03 *$ \\
\hline $\mathrm{BCP}+\mathrm{DMN}$ & 50 & $0.668 \pm 0.084 *$ & $0.253 \pm 0.135$ & $89.43 \pm 3.24 * *$ \\
\hline $\mathrm{BCS}+\mathrm{DMN}$ & 50 & $0.554 \pm 0.172$ & $0.375 \pm 0.293$ & $96.80 \pm 4.42$ \\
\hline \multicolumn{5}{|l|}{ B. kaoi roots } \\
\hline $\mathrm{BKW}+\mathrm{DMN}$ & 100 & $0.695 \pm 0.097 *$ & $0.214 \pm 0.128$ & $87.53 \pm 3.11 * *$ \\
\hline $\mathrm{BKP}+\mathrm{DMN}$ & 50 & $0.677 \pm 0.113^{*}$ & $0.289 \pm 0.145$ & $91.02 \pm 2.60 *$ \\
\hline $\mathrm{BKS}+\mathrm{DMN}$ & 50 & $0.572 \pm 0.072$ & $0.479 \pm 0.070$ & $91.37 \pm 1.26^{*}$ \\
\hline
\end{tabular}

Each data was determined in rats liver homogenates and each value presents the mean \pm S.D. $(n=6)$. DMN $(10 \mathrm{mg} / \mathrm{kg}$ per day for 3 consecutive days of each week for 4 weeks $)$ was injected i.p. to induce hepatic fibrosis in rats. Test drugs were administered orally twice a day for the last 2 weeks. BCW: water extracts; BCP: polysaccharide-enriched fractions; BCS: saponin-enriched fractions which were isolated from the roots of $B$. chinense. BKW: water extracts; BKP: polysaccharide-enriched fractions; BKS: saponin-enriched fractions which were isolated from the roots of B. kaoi. ${ }^{p} p<0.05$, \# $p<0.01$ significantly different from normal control group; * $p<0.05$, $* * p<0.01$ significantly different from DMN treated group.

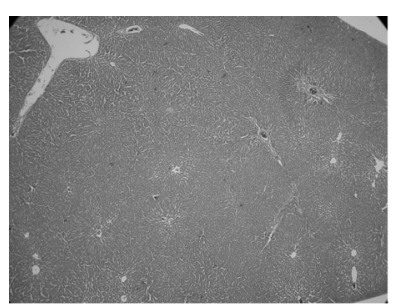

(A)

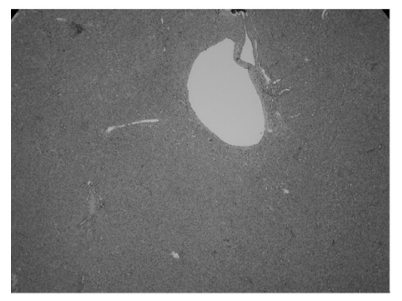

(D)

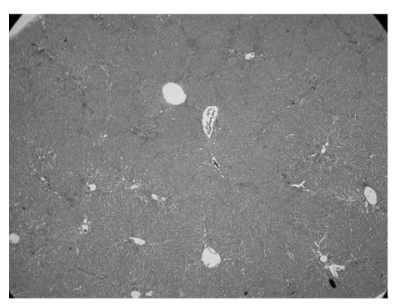

(G)

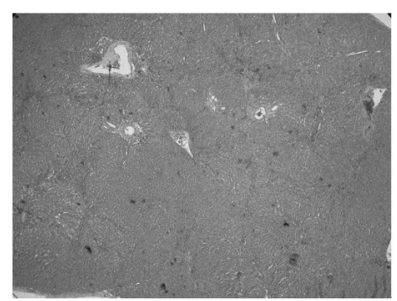

(B)

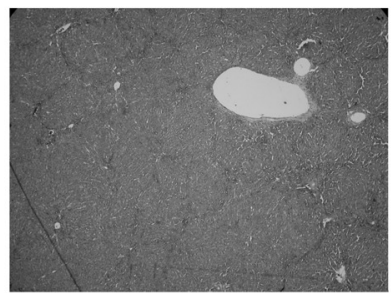

(E)

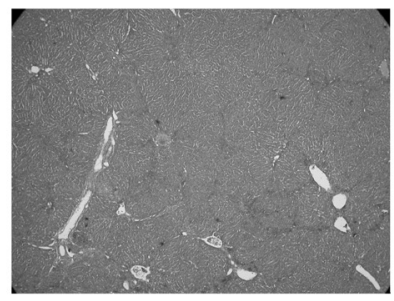

(H)

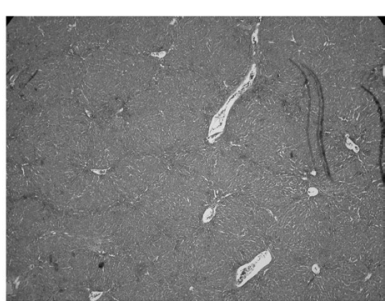

(C)

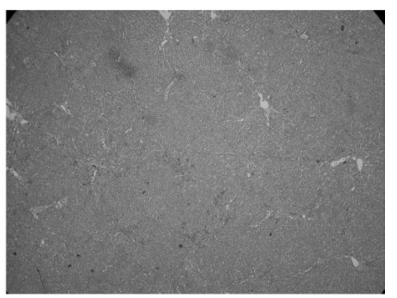

(F)

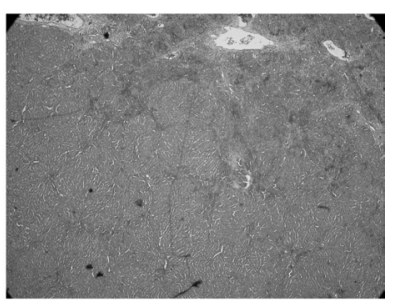

(I)

Fig. 1. The Photomicrographs $(\times 40)$ of Liver Section Taken from Rats with Hematoxylin-Eosin $(\mathrm{H}-\mathrm{E}) \mathrm{Stain}$

(A) received saline as a normal control group; (B) DMN treated group (10 mg/kg); (C) silymarin treated group (25 mg/kg) as a reference drug; (D) BCW treated group $(100 \mathrm{mg} / \mathrm{kg})$; (E) BCP treated group $(50 \mathrm{mg} / \mathrm{kg}) ;(\mathrm{F})$ BCS treated group $(50 \mathrm{mg} / \mathrm{kg}) ;(\mathrm{G})$ BKW treated group $(100 \mathrm{mg} / \mathrm{kg}) ;(\mathrm{H}) \mathrm{BKP}$ treated group $(50 \mathrm{mg} / \mathrm{kg}) ;(\mathrm{I}) \mathrm{BKS}$ treated group $(50 \mathrm{mg} / \mathrm{kg})$. Note a remarkably extensive necrosis and disruption of tissue architecture around central vein is existed in DMN treated group (Fig. 1B). These alterations were slightly or modulatory reduced with silymarin (Fig. 1C) and the two plant materials treatment in rats (Figs. 1D-I).

parenchyma. In this case, hepatic fibrosis is a common feature complicating chronic liver inflammation that eventually leads to cirrhosis. A prominent feature of hepatic fibrosis is enhanced extracellular matrix (ECM) deposition in and around the Disse's spaces (Ito cell). The ECM in hepatic fibrosis consists primary of collagen (especially collagens type
I and III). ${ }^{16,17)}$

GOT and GPT are the aminotransferase in cells especially GPT is a specific aminotransferase in liver cells. An increasing of GOT, GPT levels indicated the inflammatory stage in liver cells which chronic hepatic inflammation may lead to hepatic fibrosis. In the present study, DMN-induced increases 


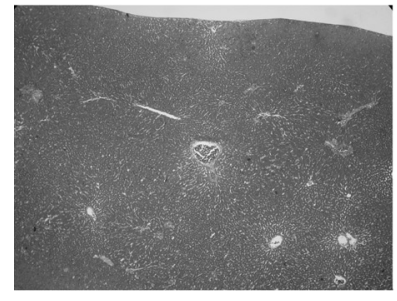

(A)

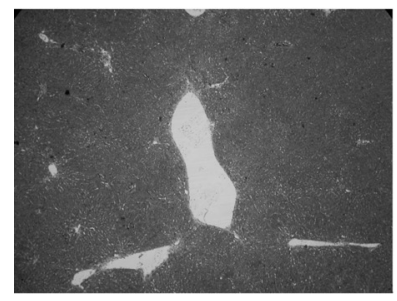

(D)

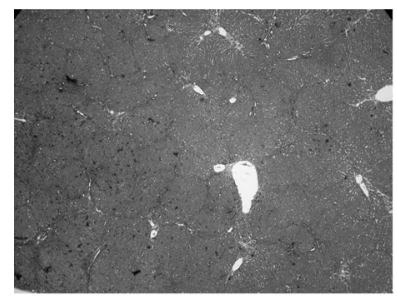

(G)

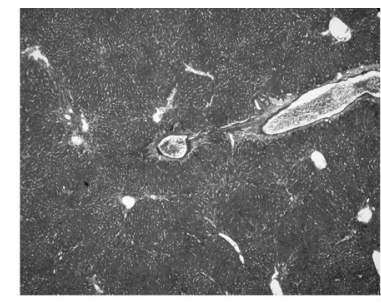

(B)

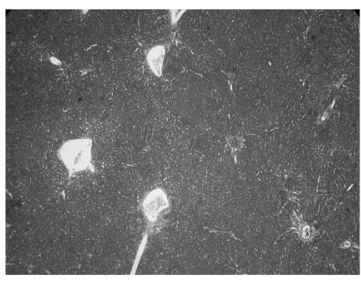

(E)

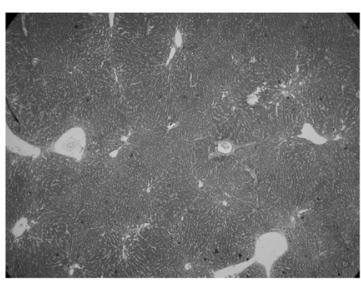

(H)

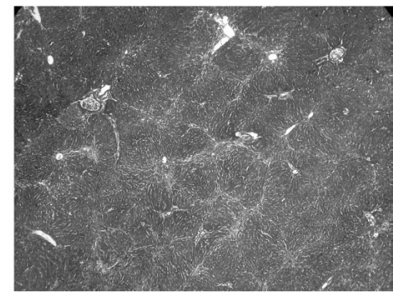

(C)

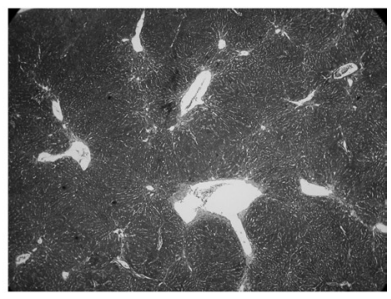

(F)

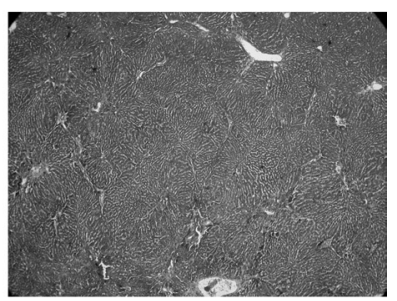

(I)

Fig. 2. The Photomicrographs $(\times 40)$ of Liver Section Taken from Rats with Masson-Trichrome (M-T) Stain

(A) received saline as a normal control group; (B) DMN treated group (10 mg/kg); (C) silymarin treated group (25 mg/kg) as a reference drug; (D) BCW treated group $(100 \mathrm{mg} / \mathrm{kg}) ;$ (E) BCP treated group $(50 \mathrm{mg} / \mathrm{kg}) ;(\mathrm{F})$ BCS treated group $(50 \mathrm{mg} / \mathrm{kg}) ;(\mathrm{G})$ BKW treated group $(100 \mathrm{mg} / \mathrm{kg}) ;(\mathrm{H}) \mathrm{BKP}$ treated group $(50 \mathrm{mg} / \mathrm{kg}) ;(\mathrm{I}) \mathrm{BKS}$ treated group $(50 \mathrm{mg} / \mathrm{kg})$. Note the liver section of the DMN treated rats exhibited an increase of collagen content, and displayed bundles of collagen fibrous surrounding the lobules, forming markedly large fibrous septa (Fig. 2B). The formation and accumulation of collagen fibers were reduced with silymarin (Fig. 2C) and the two plant materials treatment in rats (Figs. 2D-I).

serum GOT and GPT were significantly suppressed by treated with those of the two plant materials, B. kaoi and $B$. chinense, respectively. On the other hand, the in case of chronic liver diseases such as alcoholic hepatitis, hepatic fibrosis, hepatic cirrhosis et al., the serum albumin and TP levels were reduced due to protein synthesis disorder in hepatocytes. As Table 2 shows, treatment with three materials of B. kaoi significantly elevated the albumin and TP levels revealed the ability of enhancement of liver cells regeneration. According to these results of liver function examinations, however, B. kaoi showed more potent hepatoprotective effect than $B$. chinense (Newman-Keuls test).

Recently, many evidences indicated that a common link between chronic liver damage and hepatic fibrosis may be related to oxidative stress. ${ }^{18,19)}$ In addition, the free radicals produce in oxidative stress lead to biological membranes lipidperoxidation, resulting in sever cell damage and play a significant role in the pathogenesis of disease. It has been shown that certain lipidperoxidation, products induce genetic over-expression of fibrogenic cytokines and increase the synthesis of collagen ${ }^{20}$ by initiating the activation of hepatic stellate cells. $^{21)}$ Therefore, receiving free radical scavengers and reducing oxidative stress may benifit for reducing hepatic fibrosis progression. In this study, DMN-treated rats exhibited increased levels of MDA in liver homogenates which
MDA is an end product of lipidperoxidation. Neither B. kaoi nor $B$. chinense reduced the increase of MDA levels but silymarin. Briefly, silymarin belongs to flavonoids family which has been shown to possess several biological properities including hepatoprotective, anti-inflammatory and antiviral activities. $^{22)}$ A recent study indicated that naringenin, one of flavonoids, exhibited an inhibition of DMN-induced liver damage in rats. $\left.{ }^{23}\right)$ The result could explain the inhibited activity of hepatic fibrosis in silymarin may be related, partially at least, to the antioxidant and free scavenging ability.

Otherwise, GSH is widely distributed among living cells and is involved in many biological functions. It is well-established that GSH acts as an essential intracellular reducing agent for maintenance of antioxidant molecules and the thiol groups on intracellular proteins, ${ }^{24)}$ namely de $\mathrm{Ca}^{2+}$ ATPase transporter of endoplasmic reticulum. ${ }^{25)} \mathrm{GSH}$ is also the most important biomolecule protecting against chemically induced cytotoxicity, by articipating in the elimination of reactive intermediates by conjugation and hydroperoxide reduction, or of free radicals by direct quenching. ${ }^{26,27)}$ Therefore, the increased GSH content in the liver tissues of the rats may be one of the factors responsible for inhibition of lipid peroxidation. Although rats treated with BKW, BKP of $B$. kaoi and $\mathrm{BCW}, \mathrm{BCP}$ of $B$. chinense and silymarin, respectively, increased hepatic GSH levels compared to that of 
DMN treated rats, it is revealed that the anti-oxidant activity of $B$. kaoi and B. chinense may relate to the pathway of enhance GSH production.

IFN- $\gamma$ is secreted from CD ${ }^{+}$Th1 cells, $\mathrm{CD}^{+}$cells, NK cells, which plays a role in activating lymphocytes to enhance anti-microbial and anti-tumour effects. In hepatic fibrosis, IFN- $\gamma$ shows as a potent cytokine which inhibits stellate cell activation and ECM production in in vivo models of hepatic schistosomiasis, $\mathrm{CCl}_{4}$, and $\mathrm{DMN}$-induced hepatic fibrosis, respectively. ${ }^{28-31)}$ The mechanism of anti-hepatic fibrosis of IFN- $\gamma$, however, may due to the ability that IFN- $\gamma$ activates smad-7 which is able to inactive smad-2 to block downstream of tumor growth factor- $\beta$ (TGF- $\beta$ ), an indicator of profibrogenic cytokine, signal transduction. ${ }^{32)}$ In the clinical use, IFN- $\gamma$ is effective for patients with moderate chronic hepatitis $\mathrm{B}$ viral fibrosis. ${ }^{33)}$ Otherwise, DMN has been confirmed to induce IL- $1 \beta$, TNF- $\alpha$, and IL- 6 inflammatory cytokine expression in mice. ${ }^{34)}$ In immunological regulation, IL-10 is a pleiotropic cytokine that can exert either immunosuppressive or immunstimulatory effects on a variety of cell types. IL-10 has been reported to diminish secretion of TNF$\alpha$, IL- $1 \beta$, IL-6, MIP- $1 \alpha$ and MIP- $1 \beta$ after LPS challenge on neutrophils. ${ }^{35)}$ IL-10 further up-regulates IL-1 receptor expression from LPS-stimulated neutrophils. ${ }^{36}$ ) These studies suggested IL-10 played a role as an anti-inflammatory cytokines in immunomodulatory pathway. In the present study, BKW, BKP, BKS of B. kaoi and BCW of B. chinense induced higher levels of IFN- $\gamma$ more than DMN-treated rats. Moreover, BKS of B. kaoi and silymarin induced higher IL-10 concentrations more than DMN-treated rats. In contrast, silymarin was proved to be an immune-response modifier in vivo that silymarin inhibited intrahepatic expression of TNF- $\alpha$, IL-4, IL-2 and iNOS, and augmenting synthesis of IL-10. ${ }^{37)}$

In conclusion, the present study demonstrated that $B$. kaoi, an endemic plant to Taiwan, exhibited in vivo hepatoprotective and anti-fibrotic effects against DMN-induced liver injury in rats. The ability of hepatprotective and anti-fibrotic activities of $B$. kaoi are higher than $B$. chinense, a Bupleuri Radix imported from China to Taiwan. B. kaoi demonstrated the anti-inflammatory and anti-fibrotic activities followed by anti-oxidant activity of enhancement of GSH production, enhancement of liver cell regeneration and concerned with regulations of IFN- $\gamma$ and IL-10. According to the present study, we strongly suggested that $B$. kaoi should take place of $B$. chinense as Bupleuri Radix to treat chronic hepatic diseases in Chinese medicinal prescriptions.

Acknowledgements This study was supported by National Science Council of Taiwan (NSC 90-2317-B-037001), and the authors would like to thank Dr. Song-Chow Lin for his technical assistance in this experiment.

\section{REFERENCES}

1) Chiang L. C., Ng L. T., Liu L. T., Shieh D. E., Lin C. C., Planta Med., 69, 705-709 (2003).

2) Chou C. C., Pan S. L., Teng C. M., Guh J. H., Eur. J. Pharmaceut. Sci., 19, 403-412 (2003).

3) Lin S. C., Lin C. C., Lin Y. H., Supriyatna S., Pan S. L., Am. J. Chin
Med., 24, 153-164 (1996).

4) Chiu H. F., Lin C. C., Yen M. H., Wu P. S., Yang C. Y., Am. J. Chin. Med., 20, 257-264 (1992).

5) Miyamura M., Ono M., Kyotani S., Nishioka Y., J. Pharm. Pharmacol., 50, 97-105 (1998).

6) Ono M., Miyamura M., Kyotani S., Saibara T., Ohnishi S., Nishioka Y., J. Pharm. Pharmacol., 51, 1079-1084, (1999).

7) Ono M., Miyamura M., Kyotani S., Saibara T., Ohnishi S., Nishioka Y., J. Pharm. Pharmacol., 52, 111-118 (2000).

8) Lin C. C., Chiu H. F., Yen M. H., Wu C. C., Chen M. F., Am. J. Chin Med., 18, 105-112 (1990).

9) Yen M. H., Lin C. C., Chang C. H., Lin S. C., Fitoterapia, 65, 409417 (1994).

10) Yen M. H., Lin C. C., Chang C. H., Lin S. C., J. Ethnopharm., 34, $155-165$ (1991).

11) Kusunose M., Qiu B., Cui T., Hamada A., Yoshioka S., Ono M., Miyamura M., Kyotani S., Nishioka Y., Biol. Pharm. Bull., 25, 1417-1421 (2002).

12) Weng H. L., Cai W. M., Liu R. H., World J. Gastroenterol., 7, 42-48 (2001).

13) Kawai S., Kasashima K., J. Chromatogr., 495, 235-238 (1989).

14) Tietze F., Anal. Biochem., 27, 502-522 (1969).

15) Jimenez W., Pares A., Caballeria J., Heredia D., Bruguera M., Torres M., Rojkind M., Rodes J., Hepatology, 5, 815-818 (1985).

16) George J., Roulot D., Koteliansky V. E., Bissel D. M., Proc. Natl. Acad. Sci. U.S.A., 96, 12719-12724 (1999).

17) Li D., Friedman S. L., J. Gastroenterol. Hepatol., 14, 618-633 (1999).

18) Peres W., Tunon M. J., Collado P. S., Herrmann S., Marroni N., Gonzalez-Gallego J., J. Hepatol., 33, 742-750 (2000).

19) Vendemiale G., Grattagliano I., Caruso M. L., Serviddio G., Valentini A. M., Pirrelli M., Altomare E., Toxicol. Appl. Pharmacol., 175, $130-139$ (2001)

20) Parola M., Pinzani M., Casini A., Albano E., Poli G., Gentilini A., Gentilini P., Dianzani M. V., Biochem. Biophys. Res. Commun., 194, 1044-1050 (1993).

21) Parola M., Robino G., J. Hepatol., 35, 297-306 (2001).

22) Saija A., Scalese M., Lanza M., Marzullo D., Bonina F., Castelli F., Free Rad. Biol. Med., 19, 481- 486 (1995).

23) Lee M. H., Yoon S., Moon J. O., Biol. Pharm. Bull., 27, 72-76 (2004).

24) Hwang J. M., Wang C. J., Chou F. P., Tseng T. H., Hsieh Y. S., Lin W. L., Chu C.-Y., Arch. Toxicol., 76, $664-670$ (2002).

25) Hoyal C. R., Thomas A. P., Forman H. J., J. Biol. Chem., 271, 2920529210 (1996).

26) Jewell S. A., Di Monte D., Richelmi P., Bellomo G., Orrenius S., J. Biochem. Toxicol., 1, 13-22 (1986).

27) Liu C. L., Wang J. M., Chu C. Y., Cheng M. T., Tseng T. H., Food Chem. Toxicol., 40, 635-641 (2002).

28) Rockey D. C., Maher J. J., Jarnagin W. R., Gabbiani G., Friedman S. L., Hepatology, 16, 776-784 (1992).

29) Czaja M. J., Weiner R. F., Takahishi S., Giambrone M. A., Van der Miede P. H., Schellekens H., Biempica L., Hepatology, 10, 795-800 (1989).

30) Rockey D. C., Chung J. J., J. Investig. Med., 42, 660-670 (1994).

31) Baroni G. S., D’Ambrosio L., Curto P., Casini A., Mancini R., Jezequel A. M., Benedetti A., Hepatology, 23, 1189-1199 (1996).

32) Soto P., Price-Schiavi S. A., Carraway K. L., J. Biol. Chem., 278, 20338-20344 (2003).

33) Weng H. L., Cai W. M., Liu R. H., World J. Gastroenterol., 7, 42-48 (2001).

34) Schook L. B., Lockwood J. F., Yang S. D., Myers M. J., Toxicol. Appl. Pharmacol., 116, 110-116 (1992).

35) Cassatella M. A., Meda L., Bonora S., Ceska M., Constantin G., J. Exp. Med., 178, 2207-2211 (1993).

36) Cassatella M. A., Meda L., Gasperini S., Calzetti F., Bonora S., J. Exp. Med., 179, 1695-1699 (1994).

37) Schumann J., Prockl J., Kiemer A. K., Vollmar A. M., Bang R., Tiegs G., J.Hepatol., 39, 333-340 (2003). 